\title{
Gradhiva
}

GRADHIV

Revue d'anthropologie et d'histoire des arts

$24 \mid 2016$

ARTchives

\section{Suite : Gradhiva au musée du quai Branly}

Entretien avec Anne-Christine Taylor, réalisé à Paris le 31 août 2016

Anne-Christine Taylor et Giordana Charuty

\section{(2) OpenEdition}

Journals

Édition électronique

URL : http://journals.openedition.org/gradhiva/3298

DOI : 10.4000/gradhiva.3298

ISSN : 1760-849X

Éditeur

Musée du quai Branly Jacques Chirac

Édition imprimée

Date de publication : 7 décembre 2016

Pagination : 228-243

ISBN : 978-2-35744-094-4

ISSN : 0764-8928

Référence électronique

Anne-Christine Taylor et Giordana Charuty, « Suite : Gradhiva au musée du quai Branly », Gradhiva [En

ligne], 24 | 2016, mis en ligne le 07 décembre 2019, consulté le 22 avril 2019. URL : http://

journals.openedition.org/gradhiva/3298; DOI : 10.4000/gradhiva.3298

(c) musée du quai Branly 


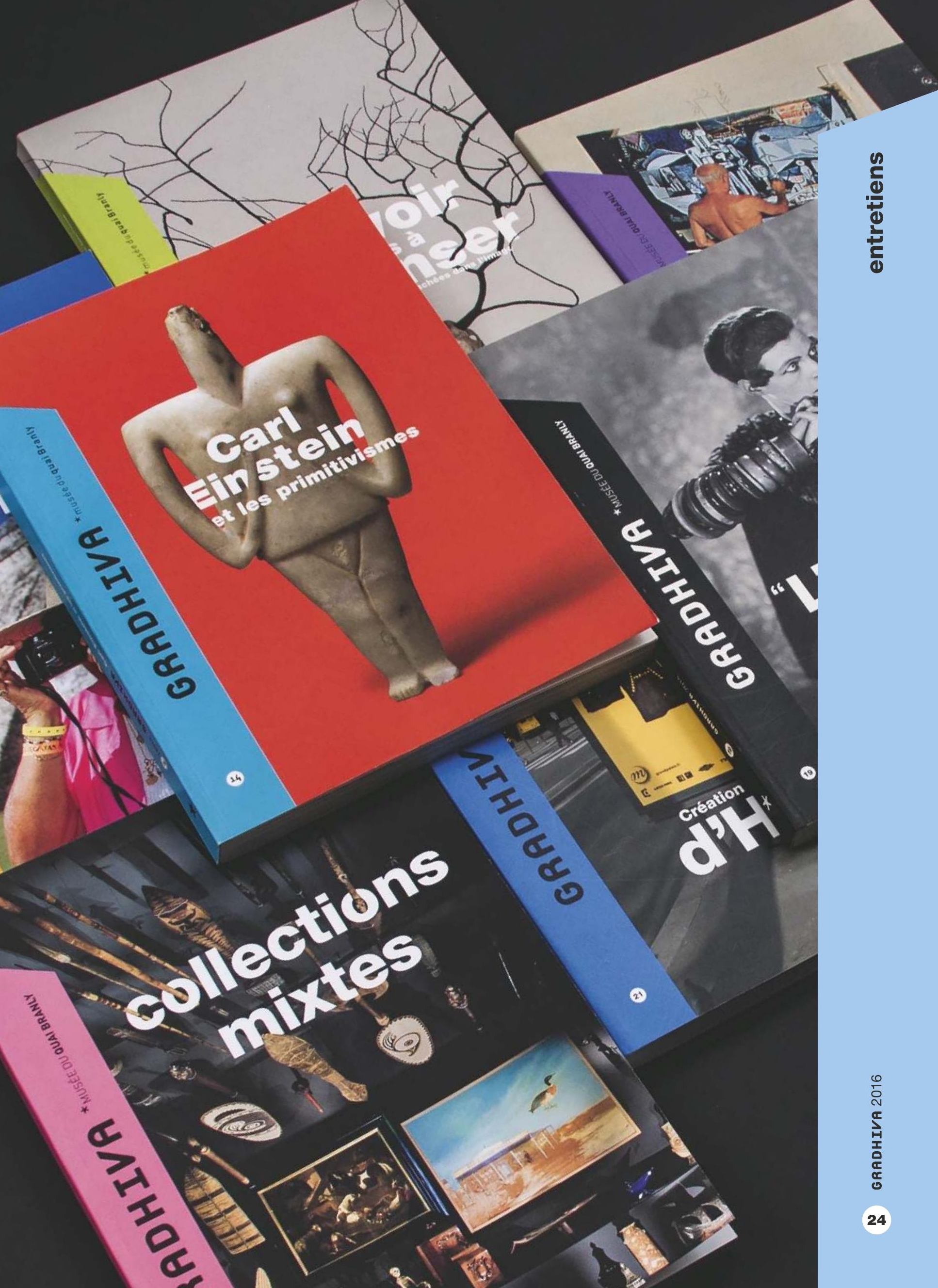




\section{Suite: Gradhiva au musée du quai Branly Entretien avec Anne-Christine Taylor ${ }^{1}$}

par Giordana Charuty

Américaniste spécialiste des cultures indigènes de l'Amazonie, directrice de recherche au CNRS, Anne-Christine Taylor a été détachée de 2005 à 2013 au musée du quai Branly, où elle a dirigé le département de la recherche et de l'enseignement. Membre d'office du comité de rédaction de Gradhiva, elle en assure la codirection depuis 2009. Elle est aussi membre des conseils de rédaction du Journal de la Société des américanistes et de la revue Terrain. Européaniste, directrice d'études à l'École pratique des hautes études (EPHE), Giordana Charuty est membre du comité de rédaction de Gradhiva au musée du quai Branly depuis 2009. 
2. Gradhiva 34 , La TraceLe Pas, avril 2004

3. Gradhiva 1, "Haïti et l'anthropologie», Carlo Avierl Célius (dir.), 2005 Le conseil de rédaction était composé de Christine Barthe, Dominique Baudin, Michèle Coquet, Brigitte Derlon, Emmanuel Désveaux, Erwan Dianteill, Serge Gruzinski, Yves Le Fur, Muriel Rausch, Anne-Christine Taylor.

4. Gradhiva 2, Salvatore D'Onofrio (dir.), «Autour de Lucien Sebag », 2005.

5. Gradhiva 3, «Du Far West au Louvre: le musée indien de George Catlin ", 2006. Dossier coordonné par Claude Macherel et présenté par Daniel Fabre.

6. Américaniste, directeur d'études à l'EHESS, Emmanuel Désveaux a été directeur scientifique du musée du quai Branly de 2001 à 2006.
Giordana Charuty - Comment s'est opéré le transfert de Gradhiva au musée du quai Branly? Comment est-on passé d'une revue d'histoire de l'anthropologie à une revue d'anthropologie des arts?

Anne-Christine Taylor - La revue a été donnée par Jean Jamin au musée en 2004 après le numéro $34^{2}$, le dernier de la série édité chez Jean-Michel Place. À ce moment-là, elle s'appelle encore «Revue d'histoire et d'archives de l'anthropologie».

G.C. - Lorsqu'elle reparaît en 2005, elle a pour sous-titre "revue d'anthropologie et de muséologie", mais il me semble qu'il y a eu une interruption?

A.-C. T. - Oui, pendant près d'un an. La revue a d'abord été gérée par la Direction du développement culturel (DDC), autrement dit par le service de production des expositions et autres manifestations culturelles du musée (concerts, pièces de théâtre, etc.). Je me souviens d'avoir assisté aux toutes premières réunions qui étaient présidées par Hélène Fulgence, directrice de ce service; Françoise Zonabend restait cependant directrice de la rédaction. Le premier numéro de la nouvelle série était le volumineux numéro consacré à Haïti ${ }^{3}$. Et le graphisme du titre accentuait non plus le « $\mathrm{H}$ », pour «histoire», mais la dernière syllabe, "VA», sans que l'on comprenne bien pourquoi... Le numéro suivant était consacré à Lucien Sebag, avec un ensemble d'articles qui relevaient de l'histoire de l'ethnologie; on restait donc tout à fait dans la continuité ${ }^{4}$. Avec le numéro $3^{5}$, on a eu, pour la première fois, une couverture et un cahier couleur; le reste était en blanc et noir et la maquette demeurait similaire. Puis le musée a décidé de confier la gestion de la revue au département de l'enseignement et de la recherche, tout en laissant au service des éditions de la DDC le soin de s'occuper de la vente et de la diffusion. Cependant, ce service avait déjà beaucoup à faire avec la production des catalogues, puisqu'il y avait à l'époque douze expositions par an et un catalogue pour chacune; du coup, il n'avait guère le temps de se soucier de Gradhiva.

G.C. - Vous étiez alors à la fois au comité de rédaction et à la direction du département de l'enseignement et de la recherche?

A.-C. T. - Oui, le moment où la revue a été rattachée à la direction de la recherche a coïncidé avec celui où j'ai pris la succession d'Emmanuel Désveaux ${ }^{6}$ en 2005 pour diriger le département. J'étais donc d'office au comité de rédaction puisque j'avais la responsabilité de la revue. Mais Françoise Zonabend est restée rédactrice en chef pendant encore un an et demi.

G.C. - L'événement, alors, c'est, bien sûr, l'inauguration en juin 2006 du musée du quai Branly, accompagnée par le numéro trois de Gradhiva sur ce qu'avait été, un siècle et demi 
auparavant, la rencontre du public parisien - surtout d'un groupe de I'avant-garde romantique - avec I'Indian Gallery de George Catlin. Comment s'est fait le choix de ce thème?

A.-C. T. - C'est Daniel Fabre ${ }^{7}$ qui l'a proposé; il préparait avec Claude Macherel et des membres de son équipe un dossier sur Catlin et pensait que le musée pouvait être intéressé. Le sujet était d'autant plus pertinent que le musée possède dix des portraits d'Indiens iowas peints à Paris par Catlin'8 et que l'on venait de découvrir des documents inédits sur la visite des Indiens à Paris. Nous avons pu, en particulier, avoir copie des croquis d'Eugène Delacroix identifiés dans son Carnet des Pyrénées grâce à Françoise Viatte, responsable du département des arts graphiques au Louvre et épouse de Germain Viatte, directeur du patrimoine et des collections au musée du quai Branly.

G.C. - En effet, il y avait depuis 2004 un "atelier Catlin" au Lahic pour combler ce qui apparaissait comme une lacune dans sa redécouverte par les historiens de l'art américain. La grande exposition au Smithsonian Art Museum, à Washington ${ }^{9}$, faisait peu de place à la tournée européenne du musée indien de Catlin, la première entreprise ethnographique exposée à Paris, avec des portraits, une collection d'objets et des représentations dansées. Or cette tournée avait produit un véritable choc esthétique sur les artistes postromantiques. Françoise Viatte a appuyé la proposition d'acquisition du “carnet pyrénéen" de Delacroix et Claude Macherel a réalisé un entretien avec Arlette Sérullaz, au Louvre, sur les croquis d'Indiens ojibwas qui figurent dans ce carnet ${ }^{10}$.

C'était très cohérent de rétablir la mémoire d'un moment complètement oublié en France, d'intense réflexion des artistes sur la "différence indienne" et sur la naissance de l'art, pour accompagner l'ouverture d'un musée qui se voulait d'“art premier"...

A.-C. T. - Par ailleurs, le thème de ce dossier Catlin correspondait parfaitement à la logique de l'exposition d'ouverture «D'un regard l'Autre ${ }^{\mathbf{1 1}}$ » et, plus généralement, à ce qu'on imaginait être, ou ce que devait être, l'esprit du musée. Au fond, c'était le premier vrai numéro de Gradhiva au musée, puisque «Haïti et l'anthropologie » et celui sur Lucien Sebag respectaient parfaitement l'esprit de la première ligne éditoriale de la revue axée sur l'histoire de l'anthropologie.

G.C. - Cependant, Gradhiva, dès sa création, affirmait que l'on ne pouvait faire l'histoire de l'ethnologie sans prendre en compte ses liens avec la littérature, la musique et les arts plastiques. Et, de fait, ce premier numéro consacré à Haïti n'accorde-t-il pas déjà une place centrale à la question des arts?
7. Directeur d'études à l'EHESS, Daniel Fabre (1947-2016) est le fondateur, en 2001, du Laboratoire d'anthropologie et d'histoire de l'institution de la culture - Lahic (EHESS-CNRS) avec une convention du ministère de la Culture. Voir Giordana Charuty, Michèle Coquet, Jean Jamin, «In memoriam. Monsieur Fabre n'est jamais là ", Gradhiva 23, 2016: 2-25.

8. II s'agit des portraits de Wa-ta-we-buck-a-nak ("Général commandant »), Ee-ah-Sa-Pa ("La Roche noire"), Say-say-Gon ("Orage de grêle"), Wan-ee-ton ("Chef des Susseton, bande des Sioux"), Shon-ta-yi-ga («Petit-Loup»), Máh-totóh-pa («Quatre ours en tenue d'apparat »), Ah-Wun-ne-wah-be ("Oiseau tonnerre»), Mu-ho-she-kaw ("Nuage blanc"), Mah-te-he-ha ("Vieil ours") et de Maungwa-daus ("Grand héros »), sur commande du roi Louis-Philippe, suite à la représentation donnée par les lowas au Louvre en avril 1845. Sur la réception de ces portraits au salon de 1846, voir Daniel Fabre, "L'effet Catlin. Paris, 1845-1846 ", Gradhiva 3 2006 : 55-75.

9. Voir George Gurney, Therese Thau Heyman (dir.), George Catlin and his Indian Gallery, catalogue d'exposition, Washington, Smithsonian American Art Museum/New York, W. W. Norton, 2002.
10. "Quand Delacroix croqua-t-il les Ojibwas? Un entretien d'Arlette Sérullaz avec Claude Macherel ", Gradhiva 3, 2006: 105-109.

11. "D'un regard l'Autre. Histoire des regards européens sur l'Afrique, l'Amérique et l'Océanie", commissaire Yves Le Fur, 19 septembre 2006 21 janvier 2007. Catalogue d'exposition: Yves Le Fur et al., Paris, musée du quai Branly/RMN, 2006. 
12. Dossier "Sismographie des terreurs ", coordonné et présenté par Jackie Assayag, Gradhiva 5, 2007. ci-contre

à gauche

fig. 1

Couverture de Gradhiva 3,

Du Far West au Louvre:

le musée indien de George Catlin. Paris, musée du

quai Branly, 2006.

ci-contre

à droite

fig. 2

Couverture de Gradhiva 9, Arts de l'enfance, enfances de l'art. Paris, musée du quai Branly, 2009.
A.-C. T. - C'est vrai, mais pour une raison liée à ce qu'est Haïti : la question des arts est un facteur essentiel dans la vie culturelle et économique de l'île. II était donc normal que l'art soit déjà très présent dans ce dossier. Pour revenir à la revue produite au musée, ce qu'elle devait être constituait un enjeu où entraient des considérations d'équilibre interne à l'institution. Certes, la revue avait été confiée au département de la recherche et de l'enseignement, en raison de son passé et parce que c'était une revue au carrefour de plusieurs disciplines - ethnologie, histoire, sociologie, histoire de l'art, esthétique... Le département du patrimoine et les conservateurs n'avaient rien contre la revue, mais souhaitaient qu'elle soit, d'une manière ou d'une autre, liée à leurs propres sphères d'activité. Certains voulaient que la revue devienne un équivalent plus moderne de celle du musée de l'Homme - Objets et mondes -, qui présenterait l'état du savoir et de la recherche sur les collections. Le lien avec les collections leur paraissait essentiel. Les débats sur l'orientation éditoriale de Gradhiva au musée reflétaient les tensions - d'ailleurs moins acrimonieuses que productives - entre la recherche et le patrimoine autour de la place, des moyens et des formes d'interaction entre les deux départements piliers du musée pour s'accorder sur ce que devaient être à la fois la revue et l'anthropologie au musée. D'où le sous-titre finalement adopté: «Revue d'anthropologie et de muséologie».

G.C. - Les controverses qui ont accompagné la création du musée du quai Branly et du Musée des civilisations de l'Europe et de la Méditerranée (MuCEM) à Marseille témoignaient d'un nouvel intérêt du public en général, et des anthropologues en particulier, pour ce que devait être, ou ne pas être, un musée dédié à la diversité des cultures, ou plutôt, comme le soulignait l'exposition d'ouverture, à l'histoire des regards portés sur elles. Les sciences sociales faisaient une large place à l'histoire de la muséification - le numéro 5 de Gradhiva ${ }^{12}$ traitait d'une question d'actualité, comparer les diverses manières de représenter la terreur - et la muséologie commençait à devenir une question importante...

A.-C. T. - Cela commençait. On aurait pu dire que créer une revue vraiment moderne de muséologie avait un sens en France puisque ce qui se faisait de plus intéressant dans ce domaine venait essentiellement des pays anglo-saxons. Les grandes revues de référence en matière de muséologie sont de langue anglaise; en France, elles étaient, il faut bien le dire, très poussiéreuses. Donc, cela aurait pu être. Mais une autre considération entrait en ligne de compte: quel est le public d'une revue de muséologie? Les conservateurs, mais les conservateurs des arts «traditionnels" sont peu nombreux... Par ailleurs, Stéphane Martin, le président du musée, n'a jamais voulu y développer - au département de la recherche ou ailleurs - les museum studies, estimant que l'espèce de nombrilisme muséographique que cela implique n'est pas très productif. C'était donc une voie fermée pour Gradhiva. Voilà donc où nous en étions: des problèmes de positionnement se posaient au sein même du musée et, à mon sens, de façon plus générale par rapport au paysage des revues scientifiques de langue française dans son ensemble, car Gradhiva avait perdu un peu de ses traits distinctifs 

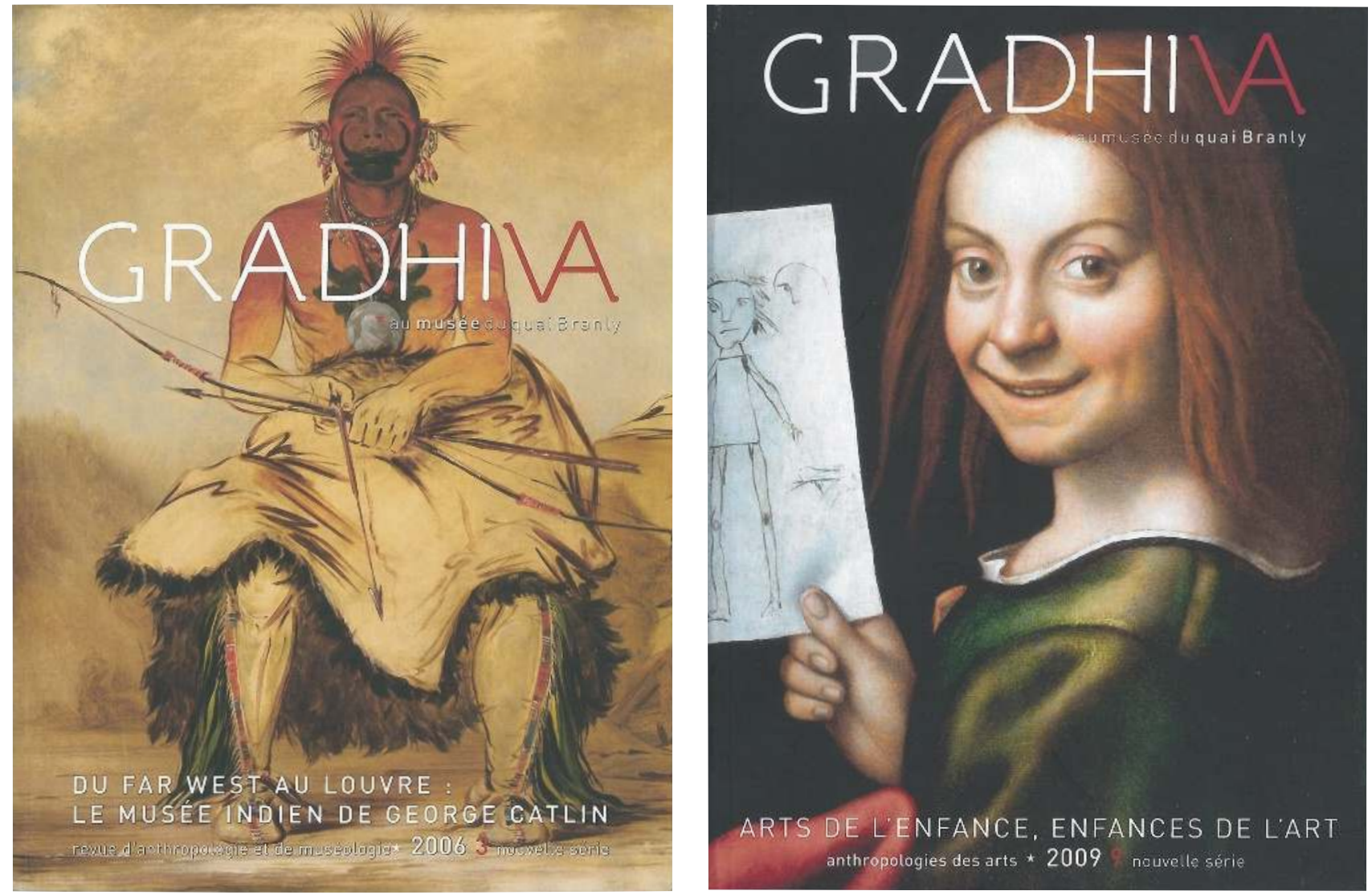
13. Voir notamment les articles suivants publiés dans les premiers numéros: André Schaeffner, «Musique savante, musique populaire, musique nationale ", Gradhiva 6 , 1989: 68-88; Sally Price, "Arts primitifs et regards civilisés ", Gradhiva 4 1988: 19-27; Sally

Price et Jean Jamin, "Entretien avec Michel Leiris », Gradhiva 4: 29 56 ; Clémentine Deliss, "Notes pour Documents. Quelques réflexions sur l'exotisme et l'érotisme en France pendant les années trente ", Gradhiva 2, 1987 : 68-73; Philippe Peltier, "Paris-Nouvelle-Guinée, 1925-1935. Jacques Viot, les Maro de Tobati et la peinture moderne", Gradhiva 8, 1990: 38-64.

14. Carlo Severi, "Anthropologie de l'art", in Pierre Bonte et Michel Izard (dir.), Dictionnaire de l'ethnologie et de l'anthropologie, Paris, PUF, 1991; Michèle Coquet, "De l'anthropologie de l'art ", Journal des africanistes 65 (2) 1995 : 222-233. Voir auss la revue Terrain 32, 1999 consacrée au beau Monique Jeudy-Ballini, "Dédommager le désir » 5-20; Marie Mauzé, "L'éclat de l'haliotide»: 83-98. depuis que Jean Jamin ne s'en occupait plus. En somme, la revue souffrait d'un trouble d'identité, et on ne savait pas trop qui était responsable de la revue et ce qu'elle devait devenir.

G.C. - En 2009, s'affirme clairement la ligne éditoriale "anthropologie des arts" qui, au fond, remet au centre l'un des intérêts de la revue façon Jean Jamin ${ }^{13}$. Deux sous-titres se succèdent rapidement: "anthropologies des arts" modifié à partir de 2010 en “anthropologie et histoire des arts"...

A.-C. T. - Ces sous-titres découlent de tout un travail de définition de ce que devait être le domaine de recherche couvert par le département, travail auquel je m'étais attelée depuis mon arrivée au musée en 2005. Dès le début, je pensais que ce champ de l'anthropologie et de l'histoire des «arts» était le territoire sur lequel devait camper le musée, étant donné la nature de ses collections, son abandon du modèle du musée ethnographique encyclopédique, ses choix muséographiques, sa volonté de faire "dialoguer les cultures "... Une fois définis les grandes lignes de la politique de recherche du musée et les dispositifs institutionnels (notamment le Groupement de recherche international «anthropologie et histoire des arts ") qui allaient la porter, il paraissait cohérent d'infléchir la ligne éditoriale de la revue pour qu'elle corresponde à cette orientation. La proposition du nouveau sous-titre a donc été soumise à la présidence du musée puis validée par son conseil d'orientation scientifique.

G.C. - De plus, il existait un vide en France dans ce domaine. Il y avait certes eu, à partir du milieu des années 1980, le séminaire d'anthropologie de l'art animé par Louis Perrois et Claude-François Baudez au musée de l'Homme, une entrée “Anthropologie de l'art" signée Carlo Severi dans le Dictionnaire de l'ethnologie et de l'anthropologie et une rubrique "Arts d'Afrique" initiée par Michèle Coquet dans le Journal des africanistes ${ }^{14}$. Pourtant, dix ans plus tard, la commission du Comité national du CNRS qui s'occupe de l'ethnologie incite encore les anthropologues à se tourner vers la question des objets, du jugement esthétique, des idiomes culturels qui motivent l'expression - qu'on la dise artistique ou non - des "autres".

A.-C. T. - Tout à fait. C'était là une chance pour le musée. On a donc cherché à élaborer un plan d'action pour la recherche et l'enseignement qui définisse ce territoire de l'approche anthropologique et historique des arts au sens le plus large possible du terme, c'est-à-dire celui de la création, de la production d'œuvres soumises à des jugements esthétiques, et pas uniquement des œuvres plastiques. Cette orientation devait en outre intéresser les conservateurs et contribuer à combler l'écart entre leurs modes de recherche et ceux des «chercheurs-enseignants ", apparemment divergents. Toutefois, si certains conservateurs étaient sensibles à cette "ouverture ", d'autres estimaient que les formes de recherche qu'on mettait en place, et que la revue reflétait, restaient trop éloignées de leurs pratiques 
et de leurs préoccupations. Pour en revenir au changement de sous-titre, si le domaine auquel il se réfère reste très large, c'est bien l'approche ethnographique des pratiques de création, de conception et de traitement des œuvres faisant l'objet d'un jugement esthétique qui est au cœur de la ligne éditoriale choisie.

G.C. - C'est, en effet, une définition très large puisque Gradhiva a publié des dossiers aussi bien sur les conceptions vernaculaires du copyright en matière musicale que sur les ingrédients nécessaires pour disposer d'un "cosmos" ou sur les politiques patrimoniales de la Chine. Et il a parfois fallu faire le détour par la fiction pour identifier les représentations de la création qui traversent les vies des créateurs. Pourriez-vous rappeler comment fonctionne la revue sur le plan technique?

A.-C. T. - Il y a eu un certain nombre de changements. D'abord, quand le département de la recherche a hérité de la revue, j'avais en tout et pour tout, en termes de personnel, une secrétaire et deux assistantes. Julie Trébault s'occupait avec moi de tout ce qui était mise en place des dispositifs; et Sophie Leclercq, qui avait un doctorat d'histoire culturelle (elle a fait une très belle thèse, depuis publiée, sur le surréalisme ${ }^{\mathbf{1 5}}$ ), était chargée de Gradhiva à temps partiel. Lorsqu'elle a quitté la direction de la revue en 2007, Françoise Zonabend a proposé Erwan Diantiell, à l'époque à l'École des hautes études en sciences sociales (EHESS), pour la remplacer. C'est lui qui a favorisé la venue de Maïra Muchnik, en poste à mi-temps. Puis Sophie a quitté Paris et Maïra, qui a aussi une formation universitaire de haut niveau en anthropologie (sa thèse porte sur les cultes de possession en Argentine $\left.{ }^{\mathbf{1 6}}\right)$, est devenue secrétaire de rédaction.

G.C. - Cependant, au niveau du contenu, on revenait plutôt à une ligne éditoriale d'avant Catlin puisqu'on reprenait des thèmes classiques comme les transformations de la possession dans un monde globalisé ou bien la question des mises en mémoire...

A.-C. T. - Tout à fait. Le numéro sur la possession ${ }^{\mathbf{1 7}}$ relevait, ce qui était normal, des intérêts d'Erwan Dianteill, et le comité de rédaction renouvelé était redevenu celui d'une revue d'anthropologie générale ${ }^{\mathbf{1 8}}$. On est alors en 2006-2008, avant que la revue ne prenne une direction plus claire. Ce qui la caractérisait, c'était l'importance de l'iconographie, essentiellement illustrative, Erwan Dianteill tenant beaucoup à ce que les illustrations soient proposées par les ethnologues eux-mêmes; il s'agissait d'images de terrain, très documentaires, quelle que soit leur qualité visuelle.

Après le départ d'Erwan Dianteill, Stéphane Martin a souhaité que je devienne directrice de la rédaction. Mais pour des raisons d'équilibre interne au musée - puisque le problème de la place des conservateurs à la fois au département de la recherche et dans la revue restait une petite zone de tensions occasionnelles $\mathbf{1 9}^{\mathbf{1 9}}$ et, outre l'amitié que j'avais pour Daniel Fabre,
15. Sophie Leclercq, La Rançon du colonialisme: les surréalistes face aux mythes de la France coloniale (1919-1962), Paris, Les presses du réel, 2010.

16. Maïra Muchnik, Le Tango des orixás les religions afrobrésiliennes à Buenos Aires, Paris, L'Harmattan, 2006.

17. "Le possédé spectaculaire. Possession, théâtre et globalisation ", Erwan Dianteill et Bertrand Hell (dir.), Gradhiva 7, 2008.

18. Le comité de rédaction est alors composé de: Emma Aubin-Boltanski, Carmen Bermand, Marc Chemillier, Brigitte Derlon, Nélia Dias, Daniel Fabre, Serge Gruzinski, Bertrand Hell, Deborah Kapchan, Frédéric Keck, Yves Le Fur, Landry-Wilfrid Miampika, Anne-Christine Taylor, Françoise Zonabend.

19. Les tensions sont désormais amoindries. Certains conservateurs, d'ailleurs, comme André Delpuech, responsable de l'unité patrimoniale Amériques, Paz NúñezRegueiro, qui travaille dans la même unité, ou encore Philippe Peltier, responsable de l'unité patrimoniale Océanie, nous ont proposé d'excellents articles.

double page suivante

fig. 3

Mises en page intérieures de numéros de Gradhiva (2008 à 2014). Photo @ polymago. 


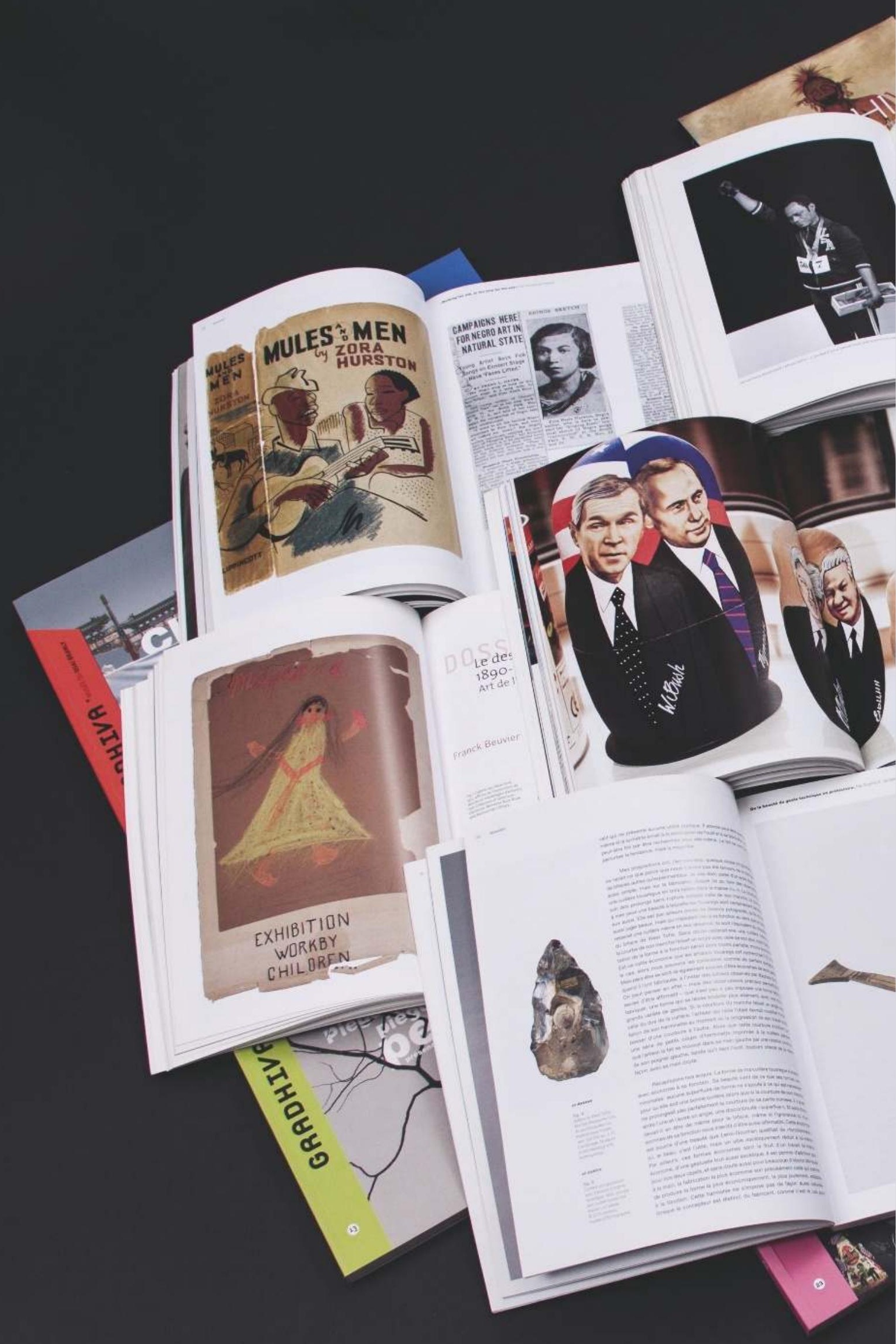




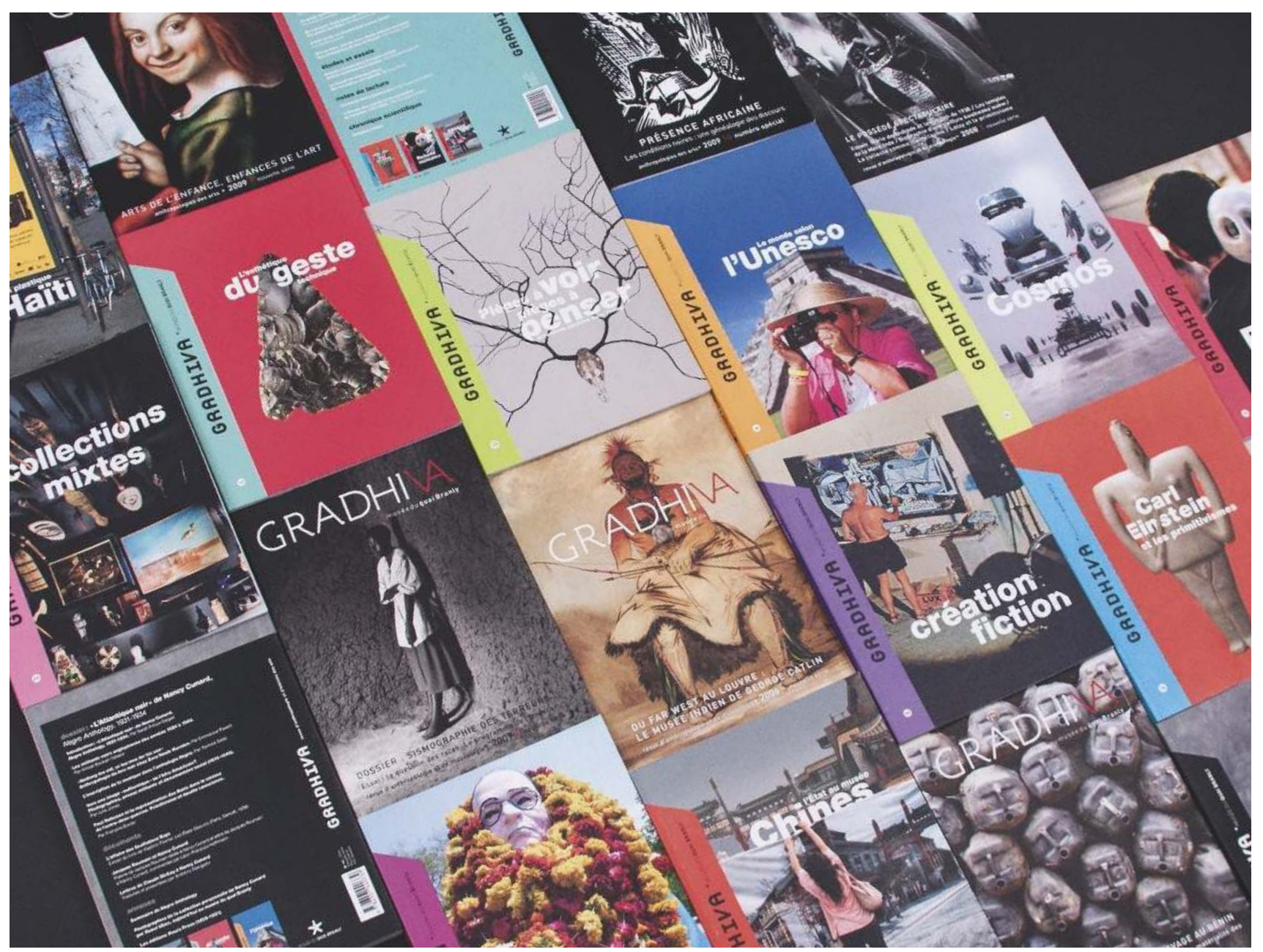


par souci d'associer l'EHESS à la revue - souci que partageait Stéphane Martin - je lui ai proposé de créer un triumvirat de direction à la rédaction, composé de moi-même, d'Yves Le Fur, directeur du département du patrimoine et des collections ${ }^{20}$, et de Daniel Fabre pour l'EHESS. L'EHESS n'a jamais mis un centime dans la revue mais, pour diverses raisons, Stéphane Martin faisait semblant de croire qu'elle était dans l'escarcelle de l'EHESS. C'était une sorte de fiction qui arrangeait tout le monde.

G.C. - Associer l'EHESS de cette façon était peut-être aussi une garantie d'audience et, en tout cas, une source importante de contributions. Le séminaire de Daniel Fabre, "L'Autre de l'art", a commencé à l'École nationale des chartes en 2002 et reprenait des questions qu'il avait traitées dans ses derniers séminaires à Toulouse: l'art populaire comme catégorie introduite par les artistes et non par les ethnographes, l'art des bergers ou encore la transformation de l'“art des fous" en "art brut" par Dubuffet, peu avant la publication de la thèse d'histoire de l'art de Lucienne Peiry' 21. Mais replacés dans la perspective d'une anthropologie des arts et d'une déconstruction de la catégorie trop générique de "primitivisme", ces objets périphériques entraient dans une problématique transversale. Cette formule "l'autre de l'art" changeait tout...

A.-C. T. - Oui, et le premier numéro du triumvirat s'est intitulé «Arts de l'enfance, enfances de l'art », avec la même maquette que le Catlin. Nous ne travaillions pas encore avec un iconographe, c'est nous qui choisissions les illustrations, les couvertures, etc. Nous demandions aux auteurs des suggestions d'images et, ensuite Sophie, Maïra et moi travaillions sur l'iconographie. En présentant la revue au conseil d'orientation scientifique, j'avais beaucoup insisté sur le fait que l'iconographie devait jouer un rôle, non pas d'illustration, de documentation, mais plutôt de contre-texte, une manière de revenir à la conception initiale de Jean Jamin, qui avait été un peu abandonnée. Ce numéro a marqué la réflexion dans ce domaine, à bien des égards.

Mais toutes les couvertures n'étaient pas du goût de Stéphane Martin. Le tableau de Caroto reproduit en couverture d'«Arts de l'enfance, enfances de l'art » l'avait en particulier heurté22. C'est pourquoi il a décidé qu'il fallait absolument changer la présentation de la revue et demandé à Nathalie Mercier, à la tête de la Direction de la communication, de proposer une nouvelle maquette, plus moderne, plus accrocheuse. L'atelier de graphistes Polymago, ayant déjà travaillé avec le musée, a alors été chargé de proposer une nouvelle maquette. Nous n'avons donc plus entièrement la main sur le choix des couvertures car les propositions de Polymago, également soumises à l'avis d'Yves Le Fur et du directeur du Département de la recherche et de l'enseignement, sont d'abord sélectionnées par Nathalie Mercier qui les montre à Stéphane Martin, auquel revient le choix final. On se trouve ainsi dans une position assez bizarre où l'on est entièrement libre quant au contenu de la revue - nous n'avons jamais eu à cet égard le moindre conflit avec le musée et Stéphane Martin ne m'a jamais fait aucun commentaire critique sur ce point - mais moins libre quant à son apparence...
20. Conservateur général, Yves Le Fur est directeur du patrimoine et des collections au musée du quai Branly. II a organisé de nombreuses expositions relatives à l'art océanien, africain et américain. Notamment: «La mort n'en saura rien, reliques d'Europe et d'Océanie " (musée des Arts d'Afrique et d'Océanie, 1999); "D'un regard l'Autre. Histoire des regards européens sur l'Afrique, l'Amérique et l'Océanie " (musée du quai Branly, 2006); « Walker Evans. Photographies" (Pavillon des sessions, Louvre, 2007); "Cheveux Chéris, frivolités et trophées" (musée du quai Branly, 2012).

21. Lucienne Peiry, L'Art brut, Paris, Flammarion, 1997

22. Giovan Francesco Caroto, Fanciullo con pupazzetto ("Garçon avec pantin»), vers 1520 . Voir le commentaire de Daniel Fabre, "C'est de l'art!" : le peuple, le primitif, l'enfant ", Gradhiva 9 , 2009: 22.

ci-contre

fig. 4

Plusieurs couvertures de numéros de Gradhiva (2006 à 2016). Photo (c) polymago. 
23. "Grands hommes vus d'en bas coordonné et présenté par Julien Bonhomme et Nicolas Jaoul, Gradhiva 11, 2010.

24. Du numéro 11 au numéro 15, Polymago a réalisé les couvertures. C'est à partir du numéro 16 que l'agence a également été chargée de créer et réaliser une nouvelle maquette pour les pages intérieures.

25. "Carl Einstein et les primitivismes", coordonné et présenté par Isabelle Kalinowski et Maria Stravinaki, Gradhiva 14, 2011 ; “"L'Atlantique noir" de Nancy Cunard. Negro Anthology 1931-1934",

coordonné et présenté par Sarah Frioux-Salgas, Gradhiva 19, 2014 ;

"Présence africaine. Les conditions noires: une généalogie des discours ", coordonné et présenté par Sarah FriouxSalgas, Gradhiva 10, 2009

26. Revue en ligne créée en 2005 à l'initiative de doctorants et de jeunes chercheurs de quatre centres de recherche installés à l'Institut national d'histoire de l'art. ci-contre

fig. 5

Couverture et pages intérieures de Gradhiva 17, L'Esthétique du geste technique. Paris, musée du quai Branly, 2013

Photo (c) polymago.

\section{G.C. - Quel est le premier numéro réalisé avec Polymago?}

A.-C. T. - “Grands hommes vus d'en bas ${ }^{\mathbf{2 3}}$ ». Nous étions tous un peu inquiets de ce qu'allait donner la nouvelle couverture, ainsi que plus $\operatorname{tard}^{\mathbf{2 4}}$ la maquette, mais il faut convenir que le résultat est très réussi. C'est aussi avec ce numéro qu'a débuté notre collaboration avec l'iconographe Valérie Loth, avec qui nous continuons à travailler. Jean Jamin lui-même avait beaucoup apprécié ce numéro et nous avait envoyé un mot. Cependant, comme il se doit au musée, la maquette des pages intérieures suit une charte graphique qu'il faut respecter à la lettre et on peut parfois regretter qu'elle soit appliquée de manière trop stricte.

Une nouvelle maquette, donc, mais fidèles aux premières heures de Gradhiva, nous avons repris le principe d'une rubrique "Documents" Iorsque cela se justifie. Par exemple dans le numéro Carl Einstein et, en général, dans les numéros qui accompagnent les expositions, comme ceux sur Nancy Cunard ou Présence africaine 25.

G.C. - Depuis 2009, Gradhiva a adopté le principe de ne pas publier des actes de colloque, plus généralement des collections de textes issus de rencontres scientifiques, mais de solliciter des propositions de dossier argumentées et clairement centrées sur une problématique. De fait, l'un des apports de la revue me semble être sa manière de problématiser une thématique, et donc de repenser un certain nombre de questions, par exemple celle du ou des primitivismes car elle est abordée par des biais auxquels les historiens de l'art ne pensent pas, à travers des figures et des périodes qu'ils ignorent.

A.-C. T. - Oui, en effet, il s'agit bien de repenser le primitivisme à la fois avec et contre, jusqu'à un certain point, l'histoire de l'art. Et on retrouve ici la question de la recherche en anthropologie des arts encore très balbutiante en France et le fait qu'il n'y avait guère de revue française qui s'intéressait à cette question. À l'échelle internationale, c'est sans doute RES: Anthropology and Aesthetics, publiée par le Peabody Museum, qui est la revue de référence; en France, la création de l'excellente revue numérique Images re-vues ${ }^{\mathbf{2 6}}$ est à peu près contemporaine de l'ouverture du musée du quai Branly, mais elle regroupe surtout des historiens de l'art et des médiévistes et reste donc très inscrite dans la tradition d'histoire anthropologique. À part ça, il n'existait pas grand-chose en France et c'était une raison supplémentaire pour que la revue soit dédiée à ce territoire-là.

G.C. - Les historiens de l'art qui travaillent régulièrement avec le musée ont-ils contribué à cette réorientation?

A.-C. T. - C'est surtout par le biais de leurs étudiants avancés que des liens se sont formés avec la communauté des historiens d'art, notamment grâce aux boursiers du musée, parmi lesquels le comité de sélection choisit toujours quelques étudiants d'histoire de l'art. Plusieurs de ces 


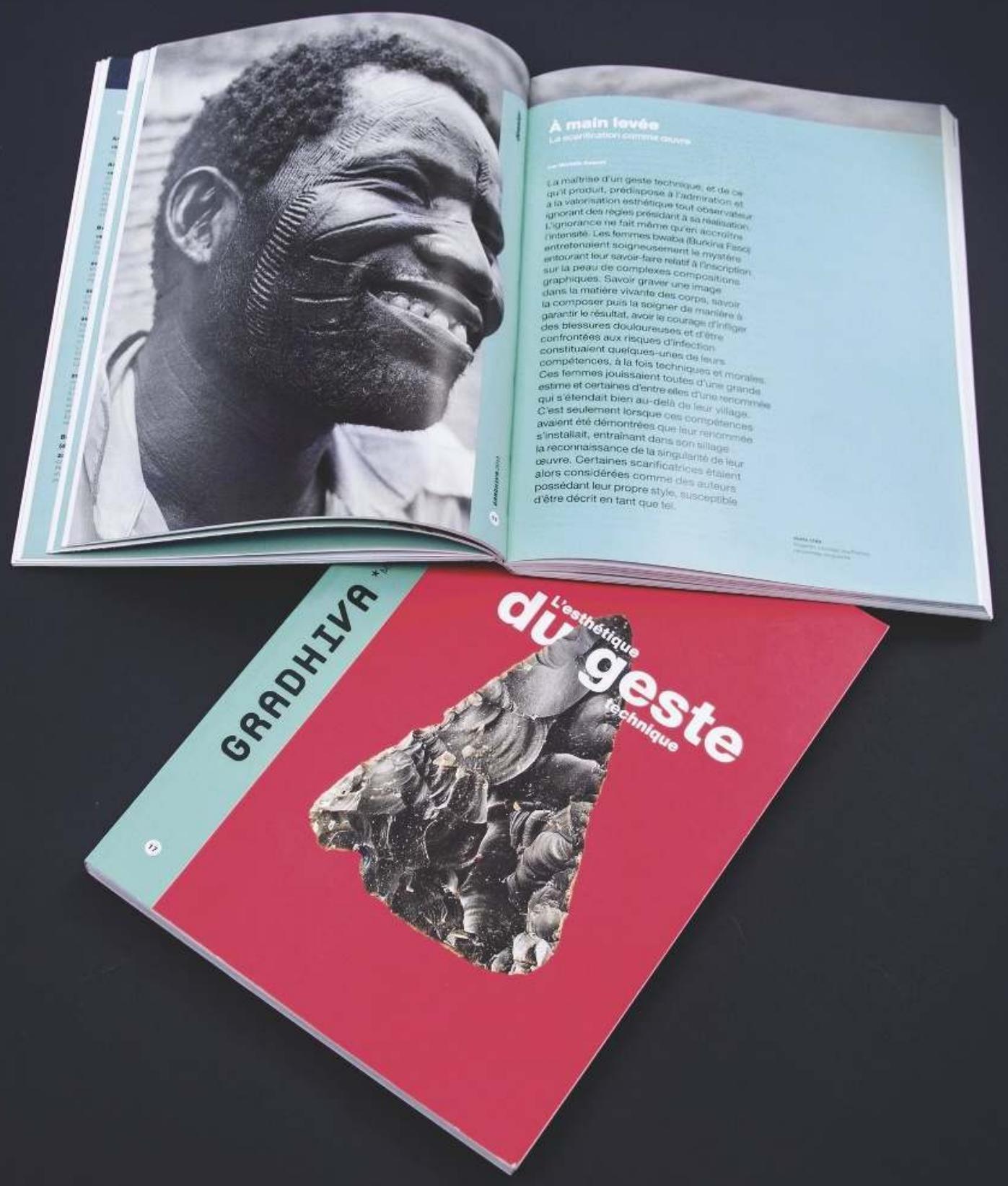


27. "La fabrique des images. Visions du monde et formes de la représentation ", commissaire Philippe

Descola, musée du quai Branly, 26 février 2010-11 juillet 2011.

28. "Pièges à voir, pièges à penser. Présences cachées dans l'image", coordonné et présenté par Carlo Severi, Gradhiva 13, 2011.

29. "L'esthétique du geste technique", Sophie A. de Beaune (dir.), Gradhiva 17, 2013. boursiers ont d'ailleurs écrit des articles pour Gradhiva. Nous avons également des liens avec les jeunes historiens de l'art actifs dans Images re-vues. Mais là on parle de la période 2009-2016; cela fait donc très peu de temps...

G.C. - Une autre grande question, qui a dialogué de manière critique avec l'exposition “La fabrique des images ${ }^{27}$ ", est celle, justement, de l’image, de la pensée visuelle, des actes d'image.

A.-C. T. - Elle est illustrée, en particulier, par le numéro qu'a coordonné Carlo Severi28, paru en 2011. C'est un domaine sur lequel on a eu peu de propositions de dossiers ces dernières années et qu'il serait bon de reprendre. Cela dit, en France, hormis Carlo Severi et Philippe Descola, peu nombreux sont ceux qui ont une vue large et théorisée des actes de vision, même si beaucoup de chercheurs s'intéressent à cette question.

G.C. - Elle a pourtant constitué à un moment donné, au sein de l'EHESS, un pôle fédérateur entre antiquisants, philosophes, historiens, historiens de l'art, anthropologues. Mais nous avons tendance à laisser cette question aux médiévistes...

A.-C. T. - Oui, par exemple des historiens comme Gil Bartholeyns (ancien boursier du musée du quai Branly) ou Pierre-Olivier Dittmar, l'un et l'autre proches des anthropologues.

G.C. - II faut souligner une autre spécificité de la revue, celle de faire redécouvrir des figures trop simplifiées ou méconnues, comme Carl Einstein dont on montre l'intérêt pour la sculpture archaïque et l'art océanien, qui élabore une anthropologie de l'image, ou Nancy Cunard, pour son rôle de "passeuse" entre plusieurs mondes et d'intellectuelle engagée dans le combat anticolonialiste.

A.-C. T. - En effet, le très beau numéro sur Carl Einstein s'écarte de l'approche habituelle des historiens d'art, par exemple celle de Liliane Meffre. Carlo Severi, lorsqu'il était encore au comité de rédaction, avait beaucoup insisté pour que le dossier suive cette voie. Pour Nancy Cunard, c'est Sarah Frioux-Salgas, responsable des archives au musée et nourrissant un intérêt particulier pour cette période, qui a proposé de faire une exposition, accompagnée d'un volume de Gradhiva riche en documents inédits ou méconnus, à l'iconographie exceptionnelle. Cette perspective sera poursuivie dans un prochain dossier, en préparation, sur Gottfried Semper.

G.C. - Le numéro sur le geste technique ${ }^{29}$ reprend, là aussi, une question classique pour la déplacer.

A.-C. T. - La question est déplacée parce que traitée par des préhistoriens et des ethnologues, ce qui défait les clichés sur l'esthétique 
entendue comme adjuvant du travail. Car c'est tout le contraire: c'est l'esthétique qui, littéralement, met en forme le travail, le geste technique. C'est une avancée qui s'inscrit dans le droit fil du travail d'André Leroi-Gourhan, en le développant de façon novatrice, notamment par l'inclusion de cas ethnographiques très soigneusement étudiés. En témoignent le bel article de Michèle Coquet sur les scarifications ou celui de Marianne Lemaire sur les chants de labour ${ }^{30}$, qui montrent bien le façonnage esthétique de chaînes d'actions techniques.

\section{G.C. - Et les robots?}

A.-C. T. - Un dossier sur les robots se justifiait notamment parce que la robotique sert de plus en plus la création de figurations de non-humains, question sur laquelle travaillent Emmanuel Grimaud, avec les automates religieux indiens, Denis Vidal et Joffrey Becker dans leur ethnographie des ingénieurs de robots ${ }^{31}$... Les questions d'esthétique jouent là encore un rôle absolument central, surtout pour les robots destinés à interagir directement avec les humains. II nous paraissait donc intéressant d'explorer cette frontière-là.

\section{G. C. - A-t-on une idée du lectorat?}

A.-C. T. - Non, sans enquête, c'est difficile. La revue est tirée à 900 exemplaires, un peu plus lorsque le numéro sert de catalogue pour une exposition, comme ceux sur Présence africaine ou Nancy Cunard. Plusieurs numéros sont épuisés au bout de deux ou trois ans. II reste la question toujours non résolue du fonds Jean-Michel Place, autrement dit le fonds de l'ancienne revue. Lorsque Jean Jamin a donné la revue au musée, les anciens numéros et, surtout, la liste des abonnés appartenaient à JeanMichel Place. Le prix initialement demandé par celui-ci pour la cession du fonds paraissait bien trop élevé. Du coup, on a perdu des abonnés. Comme la grande majorité des revues françaises en sciences sociales, Gradhiva est aujourd'hui diffusée électroniquement par Cairn.info, qui lui apporte nombre d'abonnés institutionnels. Au bout de deux ans, les numéros sont en accès libre sur le site Revues.org. Bien entendu, le travail de Flammarion, notre diffuseur, permet également d'acheter la revue au numéro en librairie.
30. Michèle Coquet "À main levée. La scarification comme œuvre", Gradhiva 17, 2013, p. 94-117; Marianne Lemaire, «Du geste technique à la geste musicale", Gradhiva 17, p. $50-69$.

31. "Robots étrangement humains ", Denis Vidal et Emmanuel Grimaud (dir.), Gradhiva 15, 2012. 